\title{
PROBLEMS AND PROSPECTS FOR DIABETES IN THE 1990s
}

\author{
A. C. MAcCUISH \\ Glasgow
}

It is appropriate to conclude this issue with a vignette of some problems and future prospects for diabetes in the last decade of the century. The reader will appreciate that coverage of the topic is selective, that the views expressed are the author's own and that references for further reading are kept to a minimum.

The first and most obvious problem is that the diabetic syndrome is common and prevalence is certainly increasing. In the Northern Hemisphere, especially in Europe, the incidence of insulin-dependent (type 1) diabetes has risen inexorably over the past decade and much evidence (discordance in identical twins, disease clustering, migration studies and variations in incidence over short time periods) indicates the clear importance of environmental factors in disease pathogenesis. For non-insulin-dependent (type 2) diabetes, it is unhappily evident that the disease is held in check only by the misery of famine in many underdeveloped countries, and even here the dietary consequences of malnutrition may induce carbohydrate intolerance. ' For First World countries, zero population growth and an ageing population guarantee the rise of non-insulin-dependent diabetes and there is no reason to believe that the World Health Organization's estimate of $3 \%$ world population prevalence of diabetes is inaccurate: indeed it may be conservative.

A second grave problem is the inaccuracy with which the gross financial and social consequences of diabetes and its treatment, especially its complications, are recorded by modern health care systems. There is now excellent evidence that diabetes as a cause of hospital admission in the United Kingdom is seriously underestimated, perhaps by $100 \%$, and accurate data are the prerequisite for proper funding and allocation of scarce resources (Fig. 1). ${ }^{2}$ Communities which have attempted formal measurement of the true costs of diabetes - the economics of a pregnancy, the rehabilitation of an amputee, provision for the blind, etc. - have been sobered by the enormity of sums involved but equally have accrued

Correspondence to: Dr. A. C. MacCuish, Diabetes Centre, Royal Infirmary, Castle Street, Glasgow G4 OSF, UK. invaluable information as to which areas merit higher investment in preventive and therapeutic measures to mitigate the impact of the disease. ${ }^{3}$

Thus a common disease is becoming more common and consumes increasing quantities of every society's health and welfare budgets. It is appropriate to consider briefly what prospects are envisaged to improve the situation and what preventive strategies may be employed.

\section{PROSPECTS FOR DIABETES: PREVENTIVE STRATEGIES}

Preventive strategies for diabetes in its broadest sense are three-layered (Table I) and will now be considered. Their classification in reverse order is not mere perversity; rather it reflects the increasing degree of difficulty in accomplishing worthy objectives as the focus moves through tertiary to primary prevention.

\section{Tertiary Preventive Strategies}

Tertiary prevention can be considered as an exercise in damage limitation and comprises all measures which may be taken to limit the consequences of long-term diabetic complications (Table II). In essence, it deals with a population of patients who have suffered from overt diabetes for 5-15 years or more and are (or will become) disabled by microvascular disease. Major morbidity in such patients is caused by retinopathy, neuropathic foot ulceration with limb loss and end-stage renal failure.

Prospects for improvement here rest almost entirely on more timely and wider use of established therapies. Detection and treatment of retinopathy at its optimal stage has been considered earlier in this issue and is of proven value in maintaining useful vision for the large majority of patients: two-thirds of those with maculopathy, more than $80 \%$ with proliferative retinal changes. For prevention and

Table I. Prospects for diabetes: preventive strategies

Tertiary prevention : Limit consequences of long-term complications Secondary prevention : Control the metabolic abnormalities Primary prevention : Reduce the incidence of disease 


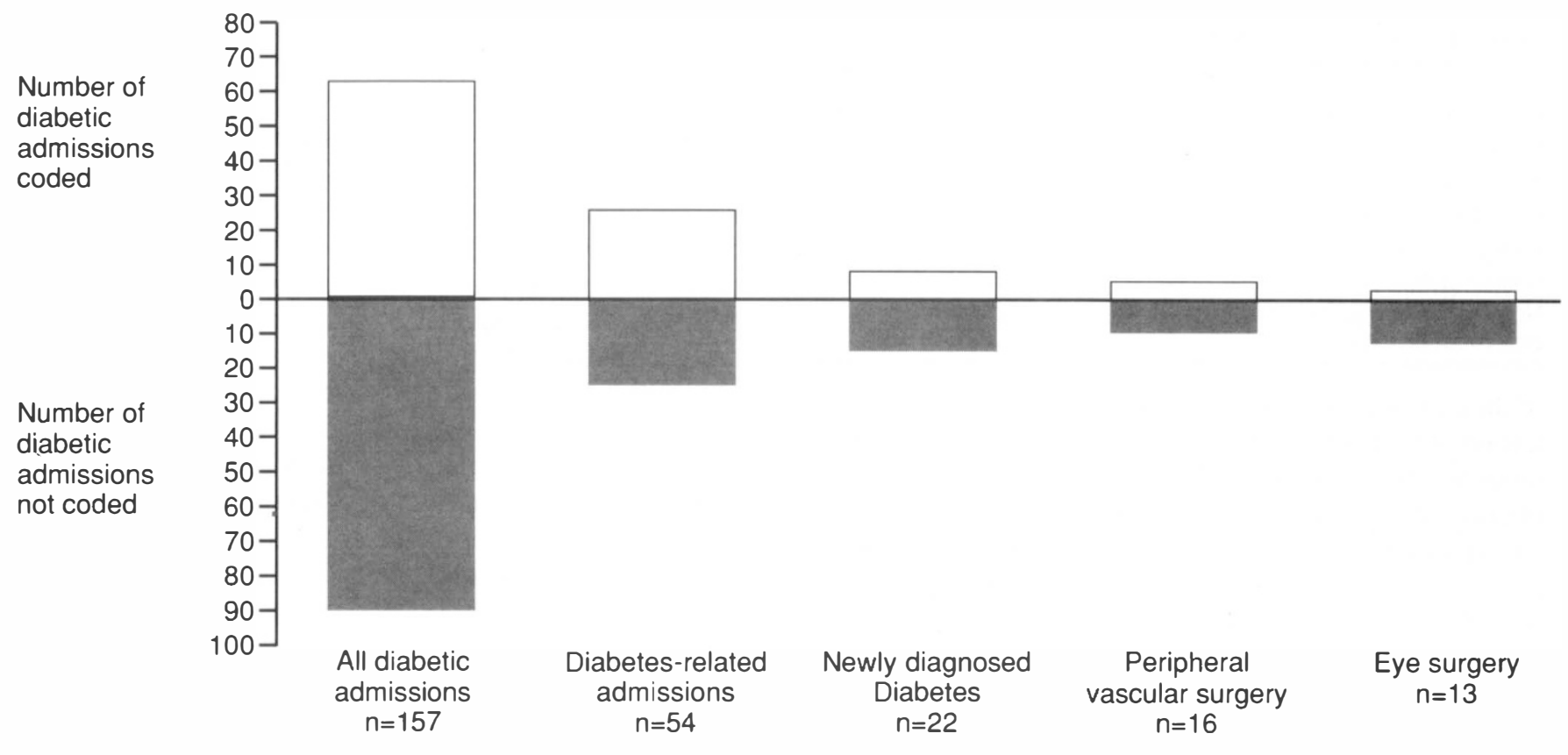

Fig. 1. The under-reporting of hospital admissions of diabetic patients using a standard morbidity coding system (WHO ICD-9). The percentage of admissions related specifically to diabetes is underestimated by more than 100\% of the true figure. (Reproduced from Leslie et al., by kind permission of the editor of Diabetic Medicine.)

treatment of foot ulceration, the process starts with proper patient education in foot care, extends through provision of a chiropody service which is readily accessible to all diabetics and concludes with the collaboration of a surgeon who has particular interest in diabetes and is expert in techniques for maintaining a vascular supply to the affected limb. Much experience has now accrued in the creation of integrated foot care service for diabetes. The benefits are so significant, without excessive allocation of new resources, that we may hope such services will steadily be developed in the regional diabetes centres.

End-stage renal failure (ESRF) remains the most complex and expensive to treat of the long-term problems, typically appearing after 15-25 years of insulin-dependent diabetes but much earlier in non-insulin-dependent diabetes. ${ }^{4}$ Haemodialysis is the longest-established therapy, but except in the United States (where two-thirds of all patients with ESRF receive this treatment) is not now regarded as the treatment of choice. Its limitations include premature death from macrovessel disease or sepsis and an exceedingly poor quality of life for many patients. It is also extremely expensive. In almost all other countries, including the United Kingdom, continuous ambulatory peritoneal dialysis (CAPD) is the treatment of choice for virtually all patients except those with severe physical dis-

Table II. Prospects for diabetes: tertiary prevention

\section{Problem}

Limit consequences of long-term complications (treatment of retinopathy, treatment of foot ulceration, renal replacement therapy)

Prospects

Detect and treat retinopathy at optimal stage

Education/chiropody/vascular surgery for foot ulceration

Better provision for cadaveric transplants (developed countries)

CAPD (developing countries) ability or visual handicap. Physical and psychological quality of life is better than for haemodialysis, intermittent heparinisation is avoided, control of hypertension and glycaemia is improved and sepsis from the intraperitoneal catheter is the only serious complication. Above all the CAPD system is effective, simple and cheap enough to be sensibly contemplated by countries with under-developed health care systems. By contrast, the treatment of ESRF by living-donor or cadaveric graft is undoubtedly the best of all therapies and, since the introduction of cyclosporin as an immunosuppressant, yields results (in graft survival) comparable to those in non-diabetics. By reasons of cost, technology and organ availability alone, transplantation is likely to remain the province of affluent countries until well into the next century.

In summary, current strategies for tertiary prevention in diabetes are remarkably satisfactory and, save for kidney transplant, not prohibitively expensive for most national health services. Our real problem is to ensure that simple measures are properly used, and we need only look at the statistics for diabetic blindness to realise that there is much scope for improvement in contemporary clinical performance.

\section{Secondary Preventive Strategies}

Secondary preventive strategies (Table III) are deployed to control the metabolic abnormalities which ensue from chronic hyperglycaemia and its treatment. In the short term, such measures are used to prevent death from uncontrolled diabetes (due to ketoacidosis and other syndromes of metabolic decompensation) or neurological damage from inadvertent over-treatment (insulin-induced hypoglycaemia). The successful treatment of metabolic decompensation rests essentially upon early recognition 
Table III. Prospects for diabetes: secondary prevention

Problem

Control the metabolic abnormalities (prevent ketoacidosis, prevent hypoglycaemia, prevent long-term complications)

Prospects

Early recognition and treatment (for ketoacidosis)

Human insulin analogues (prevent hypoglycaemia)

Control ambient glycaemia and blood pressure (prevent complications) Novel drug treatments (aldose reductase inhibitors, ACE inhibitors, etc., control complications)

of the condition and prompt treatment by careful administration of appropriate insulin, fluid and electrolyte replacement in these seriously ill patients. The principles of treating metabolic decompensation are very well established in modern practice and should pose no special problems for the trained clinician. No patients now should die from failure to correct the acidosis or potassium deficit of a common medical emergency, but the continuing mortality from ketoacidosis is a sad reminder that not all doctors are equally skilled in its management. By contrast, occasional hypoglycaemic reactions are an unavoidable fact of life for virtually all insulin-treated patients but have excited particular concern in the past 5 years, stemming from speculation that modern human insulin formulations may diminish hypoglycaemic awareness and thereby increase the risk of severe, damaging hypoglycaemia. National diabetes organisations in the United Kingdom, United States and other countries have examined this proposition seriously and with great care: the weight of expert scientific opinion has concluded that human insulin therapy carries no special risks of facilitating hypoglycaemic unawareness, ${ }^{5}$ but controversy rumbles on and (having attracted the attention of the lay press) engenders considerable anxiety for many patients. Their understandable fears can be allayed first by reassurance that the older animal insulins (bovine and porcine) are still available for clinical use, and second by knowledge that a new generation of insulins is steadily being developed. Modern techniques of recombinant DNA technology permit the insertion into host cells of synthetic genes which yield analogues of human insulin, usually by manipulation of the peptide sequence of its beta chain. Such 'designer molecules' can ensure the production of insulin formulations which will have different and more predictable action times when injected subcutaneously. The first new analogues of human insulin are already appearing for clinical trial and carry the promise of finally removing the real or imagined hazards of recombinant human insulin.

Over a longer time course, secondary prevention in diabetes is focused upon measures which may prevent or limit the complications of the disease. A convincing body of evidence now indicates that diabetic microvascular disease can be contained or minimised if ambient glycaemic levels are consistently maintained at near normal values: in practical terms, this translates to blood glucose levels below $10 \mathrm{mmol} / \mathrm{l}$ at all times. Small wonder, then, that much of the effort in modern diabetes care is directed to the goal of attaining 'good diabetic control' by careful patient education, frequently reinforced, widespread use of home blood glucose self-monitoring and use of glycated protein assays (such as HbA1) to judge glycaemia. The difficulties of achieving and maintaining good control over months and years are always formidable and are insuperable for many patients, but potential benefits are so high as to justify the commitment involved. Success or failure can be judged by regular review, usually at annual intervals, to search specifically for the presence or absence of evolving complications and detection of other factors, especially hypertension, which may accelerate their appearance.

Of course it is accepted that full correction of all the metabolic abnormalities of the diabetic state is impossible without recourse to an artificial beta cell or restoration of endogenous insulin production. The first approach (use of a Biostator or similar device) is unrealistic and impossible for almost all patients, but the latter aim - implantation of a new beta cell colony - is slowly becoming more feasible. Earlier, heroic attempts by segmental grafts of cadaver pancreas have now been superseded by the much more promising technique of transplanting small nests of cultured islet cells into the portal system. Such studies are in their infancy but islet cell transplantation has already been used successfully to treat and 'cure' a handful of adult patients with insulin-dependent diabetes and is likely to be used increasingly over the next decade.

Secondary preventive strategies must also envisage pharmacological intervention to limit or prevent the onset of microvacular disease. No single drug has yet been proven of conclusive value in this context, but at least on sound theoretical grounds it is possible that the aldose reductase inhibitors may limit the process of protein glycation in many organs and that (for example) angiotensin converting enzyme (ACE) inhibitors or aminoguanidine may check the progression of diabetic nephropathy. All such therapies are currently the subject of formal clinical trials and it is to be hoped that one or more may find a useful place in long-term diabetes management.

Finally, it is appropriate to conclude this section with the rather discouraging observation that at present we seem to have no means of containing the non-specific vascular diseases (macrovessel disease) which are the leading causes of excess mortality in diabetes. In contrast to specific microvessel disease, epidemiological evidence and population screening indicates that very modest elevations of blood glucose, well short of frank diabetes, confer a major increase in risk of morbidity and mortality from myocardial infarction, stroke and peripheral vascular disease. Even the best-controlled of diabetic patients is unlikely to attain true normoglycaemia by any means over prolonged periods and it follows that at present we can do virtually nothing to reduce excess macrovessel disease in the diabetic population.

\section{Primary Preventive Strategies}

The primary preventive strategies for diabetes are summarised simply as any means used to reduce the incidence 
of the disease. It will at once be apparent that such measures are characterised equally by extreme difficulty and high promise.

Prospects for prevention of insulin-dependent diabetes are significant and hopeful (Table IV). Much understanding of the natural history of the disease has been acquired over the past 17 years or so. We are now near the stage where the genes involved in susceptibility to insulindependent diabetes will be identified and sequenced, and before the end of the decade it will be possible to contemplate prevention of the condition by large-scale population screening of susceptible juveniles, using micromethods to screen for immunological or genetic markers in blood samples obtained by fingerprick estimation. Such a programme would be reasonable only if we could be confident of identifying the $1-3 \%$ of the population who are most susceptible to the disease, but small-scale screening studies of insulin-dependent diabetes in families do give real grounds for optimism.

A health care system committed to population screening must be matched by the prospect that safe and effective preventive therapies are possible. This is a very large caveat, and for insulin-dependent diabetes the prospects divide evenly into measures which shield susceptible persons from the environmental triggers of an autoimmune disease and therapies which check established beta cell damage. As noted earlier, environmental factors are probably of major importance in initiating the majority of cases of insulin-dependent diabetes and broadly comprise common childhood viral infections (coxsackie, mumps, rubella, etc.) or ingested dietary allergens of which the most fascinating and perhaps most common is the bovine albumin peptide found in trace quantities in cow's milk. ${ }^{6}$ Thus one approach to prevention envisages susceptible infants, identified shortly after birth, receiving a polyvalent antiviral vaccine and breast rather than bottle feeding to avoid exposure to autoimmune triggers.

For persons in whom the process of beta cell destruction has already started, up to and including the threshold of clinical diabetes, immunotherapy offers the only sensible prospect of cure. The chosen agent must perform in a difficult environment, where perhaps $85 \%$ of beta cell mass is already lost, and must be both effective and safe. No current drug meets these criteria: cyclosporin A has been used in this context and 'rescues' perhaps $20 \%$ of young persons who are given the drug at the very onset of overt diabetes. Its effects on the immune system are too nonspecific and in prolonged use it is too toxic, especially to

Table IV. Prospects for diabetes: primary prevention of insulindependent diabetes

Problem

Reduce the incidence of insulin-dependent diabetes

Prospects

Micromethods of mass screening for susceptible juveniles

Identification/elimination of environmental triggers

Safer immunomodulation (not cyclosporin)

Novel drug therapies (nicotinamide)

Specific immunotherapy the kidney, to be seriously considered as a routine treatment. Better success depends upon progress in molecular biology, and therapeutic clonotypic vaccines already being developed to treat patients with cancer have enormous potential applications for diabetes and other autoimmune diseases. ${ }^{7}$ Of equal current interest is the possible role of nicotinamide in prevention progression to insulindependent diabetes in susceptible subjects with early evidence of beta cell damage. Nicotinamide is a watersoluble group B vitamin which is effective in preventing onset of diabetes in animal models by manifold actions including free radical scavenging to reduce DNA damage, restoration of islet cell content of NAD towards normal and suppression of MHC class II antigen on islet cells. In man, the drug has been effective in preventing diabetes in persons with high levels of islet cell antibodies (ICA) and prolonging beta cell function in recently diagnosed insulin-dependent diabetes. Most seductively, it appears to be free of any serious side effects and will shortly be the centrepoint of an ambitious, international randomised placebo-controlled intervention study (ENDIT, the European Nicotinamide Diabetes Intervention Trial). In short, prospects for primary prevention of insulin-dependent diabetes are indeed exciting.

By contrast, current prospects for primary prevention of non-insulin-dependent diabetes are only the first faltering steps down a very long road (Table V). The control of simple obesity, so often the precipitating factor of the disease in middle age, still eludes us despite all manner of dietary, psychological and pharmacological intervention and all the social pressures which have generated the slimming industry of affluent societies. But it can be said with certainty that in the 1990s we have at least recognised that approximately 60 distinct genetic disorders are associated with clinical diabetes, and although many are rare it is apparent that gene mutations at many different loci can affect glucose metabolism. ${ }^{8}$ The process of penetrating this genetic maze has now started with the recent demonstration that one well-characterised form of non-insulindependent, maturity-onset diabetes of the young (MODY) is linked to a nonsense mutation of the gene on chromosome $7 p$ which codes for glucokinase. ${ }^{9}$ Beyond a doubt, the genetic basis of multiple other syndromes of non-insulin-dependent diabetes will steadily be disclosed by the march of molecular biology. An understanding of the genesis of these disorders carries also the tantalising prospect - still decades distant, hedged by ethical problems - of gene therapy by regulation, switching or manipulation.

Table V. Prospects for diabetes: primary prevention of non-insulindependent diabetes

Problem

Reduce the incidence of non-insulin-dependent diabetes

Prospects

Control of obesity

Identification of the genetic basis (e.g. abnormal glucokinase, chromosome 7p, MODY)

Gene therapy 


\section{CONCLUSIONS}

Clinical diabetes mellitus is the commonest endocrine disease in the world and embraces a large group of disorders. The incidence of insulin-dependent and noninsulin-dependent diabetes will continue to rise steadily for the rest of this century and beyond. The severe morbidity, economic and social consequences of diabetes will be identified with increasing accuracy. Tertiary preventive strategies for diabetes will improve slowly if currently available treatments are properly deployed. Strategies for secondary prevention will improve steadily, assisted by advances in recombinant DNA technology, clinical pharmacology and therapeutics. The primary prevention of insulin-dependent diabetes is about to emerge as a feasible prospect and promises incalculable benefits. The genetic basis for many of the disorders which induce non-insulindependent diabetes will be established before the end of the decade, but in the foreseeable future there are no real prospects of accomplishing a cure.

\section{REFERENCES}

1. Rao RH. Diabetes in the undernourished: coincidence or consequence? Endocr Rev 1988;9:67-87.

2. Leslie PJ, Patrick AW, Hepburn DA, et al. Hospital in-patient statistics underestimate the morbidity associated with diabetes mellitus. Diabetic Med 1992;9:379-85.

3. Minnesota Diabetes Surveillance Project. Economic cost of diabetes mellitus. Morbidity and Mortality Weekly Report 1991;40:229-31.

4. Markell MS, Friedman EA. Diabetic nephropathy: management of the end-stage patient. Diabetes Care 1992;15:1226-38.

5. Gerich JE. Unawareness of hypoglycaemia and human insulin. BMJ 1992;305:324-5.

6. Karjalainen J, Martin JM, Knip M, et al. A bovine albumin peptide as a possible trigger of insulin-dependent diabetes mellitus. N Engl J Med 1992;15:302-7.

7. Schwartz RS. Therapeutic clonotypic vaccines. N Engl J Med 1992;327:1236-7.

8. Editorial. Genetic basis of NIDDM. Lancet 1992;340:455-6.

9. Velho G, Froguel P, Clement K, et al. Primary pancreatic beta-cell secretory defect caused by mutations in glucokinase gene in kindreds of maturity onset diabetes of the young. Lancet 1992;340:447-7. 